\title{
Functional recovery assessment of spinal cord contusion model in male rats without therapeutic interventions
}

Sara Rezaei ${ }^{1}$, Salar Bakhtiari ${ }^{2}$, Khairollah Asadollahi ${ }^{3}$, Somayeh Heidarizadi ${ }^{1}$, Ardeshir Moayeri ${ }^{1}$, Monireh Azizi ${ }^{1 *}$

1. Department of Anatomy, Faculty of Medicine, Ilam University of Medical Sciences, Ilam, Iran

2. Department of Biochemistry, Faculty of Medicine, Ilam University of Medical Sciences, Ilam, Iran

3. Department of Epidemiology, Faculty of Health, Ilam University of Medical Sciences, Ilam, Iran

*Corresponding author:Tel: +98 8433336803 Fax: +98 8433336803

Address: Department of Anatomy, Faculty of Medicine, Ilam University of Medical Sciences, Ilam, Iran

E-mail: azizi.moaz@gmail.com

Received; 2016/03/25 revised; 2016/05/28 accepted; 2016/08/8

\section{Abstract}

Introduction: Spinal cord injury (SCI) is one of the most serious clinical diseases, which not only affects the patient's physical and mental status, but its effects will be spread to family and community. After severe spinal cord injury, astrocytes of the central nervous system (CNS) become reactive astrocytes, and play the main role of glial scar formation. The scar is a major obstacle to regeneration of axons in the spinal cord. However, the studies have found that over time, a spontaneous partial motor recovery is observed in animals with injury without intervention. Thus, in this study, the recovery of animals with spinal cord injury was assessed after 12 weeks.

Materials and methods: In this study, 12 adult male Wistar rats weighing approximately $265 \pm 15 \mathrm{gr}$ were used to assess spinal cord injury and randomly divided into 3 groups: normal control $(n=3)$, sham $(n=3)$, injury $(n=6)$. Healthy animals in the normal control group received no laminectomy or injury, and laminectomy with or without contusion model using weight drop in segment T10 of spinal cord were carried out in injury and sham groups, respectively. Locomotor function of animals in all groups were evaluated by BBB test at the first 48 hours per day and then weekly for 12 weeks.

Results: Comparison of the results of motor evaluation from the second week to the twelfth week of the group with injury without treatment showed a relative functional recovery as the BBB score of animals from 1.4 in the second week after the injury reached to 6.5 in the twelfth week.

Conclusion: The results indicate a spontaneous partial recovery in injured animals without intervention.

Keywords: Spinal cord injury, Glial scar, Functional recovery, Rat

\section{Introduction}

Spinal cord injury (SCI) is one of the most serious clinical diseases, which not only affects the patient's physical and mental status, but its effects will be spread to family and community.

Although deaths from this injury has been reduced to less than $5 \%$ but importantly, the main victims of this injury are healthy young people suffering from a long-term disability (1).

Complications of spinal cord injury include complete (or partial) cease of sensation below the affected area, which leads to an inability to walk, involuntary 
discharge of bladder and bowel and infertility (2). However, as the injury is at the higher area or more severe, complication is even broader and causes more limitation (such as complete paraplegia) for individual (2).

Other complications of the injury include increased muscle spasms, autonomic dysreflexia and cavity development in injured area called Syringomyelia (2).

The disability is not limited to this level, the long-term complications include secondary problems and disabilities such as bed sore, infection of urinary and respiratory tracts, kidney failure, osteoporosis, etc. Thus, it imposes many physical costs and psychological stress on the family and society (3).

There is a wide variety of SCI models in rodents including: transection, hemisection, resection and aspiration (4).

Contusion, due to vertebral fracture during mechanical events which causes a severe blow to the spinal cord, is the most common clinical model in humans, covering about $40 \%$ of patients with spinal cord injury, as a result, it is the most commonly used animal model for the study of SCI (5). The primary damages are mechanical and cause central hemorrhage and necrosis in the gray matter, loss of afferent and efferent fibers of white matter or less the cell destruction and death (6).

Biological damage is followed by mechanical damage. Neurodegeneration, free radical production, myelin sheath deletion, an increase in glutamate concentration, decreased levels of cAMP, apoptotic death and glial scarring are biological signs that may persist for months and lead to further destruction and exacerbated injury $(2,7)$.

Starting with the biological response, cytokines are secreted and inflammation is caused. Schwann cells with neutrophils, lymphocytes and macrophages travel to the site of the injury in a short time, and their anti-inflammatory and cytotoxic activity lead to injury development and create secondary injuries (8).
It was recently shown that some stem cells and progenitor cells present in ependymal canal may be recalled and recover damage with increased proliferation (9). However, the cell proliferation may not always lead to the restoration of spinal cord function and sometimes only fills the cavity (7).

Mothe and Tator (2005) stated that type of cells stimulated in response to injury is different depending on the model of injury. In the transection model of the spinal cord responses of stem/progenitor cells are locally limited to the area of the injury, while in contusion and compression models the responses of these cells are systemic mitotic responses that can be seen throughout the rostrocaudal part of spinal cord, the broader response is probably because larger areas of spinal cord tissue are affected in the area of the injury (10).

All three models of injury increase the proliferation of ependymal cells but there is a significant proliferation in lateral parts of spinal cord only in contusion and compression models (11).

Damaged axons in the spinal cord have the ability of repair and regeneration to some extent. But the maximum growth of axons is about a millimeter and this recovery is not enough to have satisfactory functional outcome and recovery $(8,9)$.

Many factors are involved in partial repair and regeneration after SCI, including the lack of growth factors, inherent weak capacity of neurons in CNS for regeneration (12), chemical repellent molecules (13), inhibitors associated with proteoglycan glial scar such as chondroitin sulfate proteoglicans (CSPGs) or myelinassociated protein (MAP) (14).

After severe damage, astrocytes are the most abundant glial cells in the central nervous system becoming reactive astrocytes and thus constituting a major component of glial scar developing a major obstacle to the restoration of axons in the spinal cord. Chondroitin sulfate proteoglicans secreted by reactive astrocytes are as the main components of 
axonal inhibitor in the extracellular matrix (ECM) (15).

The primary components of the glial scar are the remains of myelin and oligodendrocytes then activation and migration of microglia occur (48h) which is accompanied by invasion of other blood-derived macrophages (16).

Scar plays an important role by confining and surrounding the site of the injury and separating it from healthy tissue as well as giving an end to inflammatory reactions at the site of the injury, through the confinement of the injury site, on the other hand, glial scar inhibits the growth of axonal sprouts (17).

In the review of previous studies, relative functional recovery was observed in animals with injury that did not receive any treatments including the study by Verdu et al in 2003 on the spinal injury showed that BBB scores in injured group without intervention had relative motor functional recovery (18).

The study by Garcia-Alias et al (2004), also represented the spontaneous regeneration without intervention in the group of spinal cord injury of photochemical type (19).

Richter et al, 2005 in a study on SCI of posterior funiculus scratch type also expressed a limited regeneration in the group of injury without intervention (20).

Therefore, this hypothesis was strengthened that in the rat's model of spinal cord injury without treatment a relative functional recovery occurs, therefore, in this study to determine the recovery achieved, motor function of animals with SCI were studied over a period of 12 weeks after the injury.

In order to assess the motor function of animals, BBB (Basso, Beattie and Bresnahan) test was used (21). It was evaluated on a daily basis in the first 48 hours after the injury and then on a weekly basis until the end of the study.

\section{Materials and methods}

Dividing groups: In this study, 12 male Wistar rats weighing approximately $265 \pm 15$ gr were used. The animals were randomly divided into three groups:

-Normal control group ( $=3$ ) healthy animals (without laminectomy or injury)

-sham group $(\mathrm{n}=3)$ only laminectomy was performed

-injury group $(n=6)$ laminectomy + throwing a ten-gram weight from a height of $25 \mathrm{~mm}$

Spinal cord injury: Contusion model of SCI in animals was produced as previously described with slight modification (22). After anesthesia, using a mixture of ketamine / xylazine (60/6 mg / kg), shaving the hair at the back of the animal and disinfecting the site, an incision was created at the midline, muscles and lamina of the T9 vertebra were removed without damaging dura mater and then throwing a ten-gram weight from a height of $25 \mathrm{~mm}$ on the spinal cord, contusion model was created at T10 segment (Figure 1). 


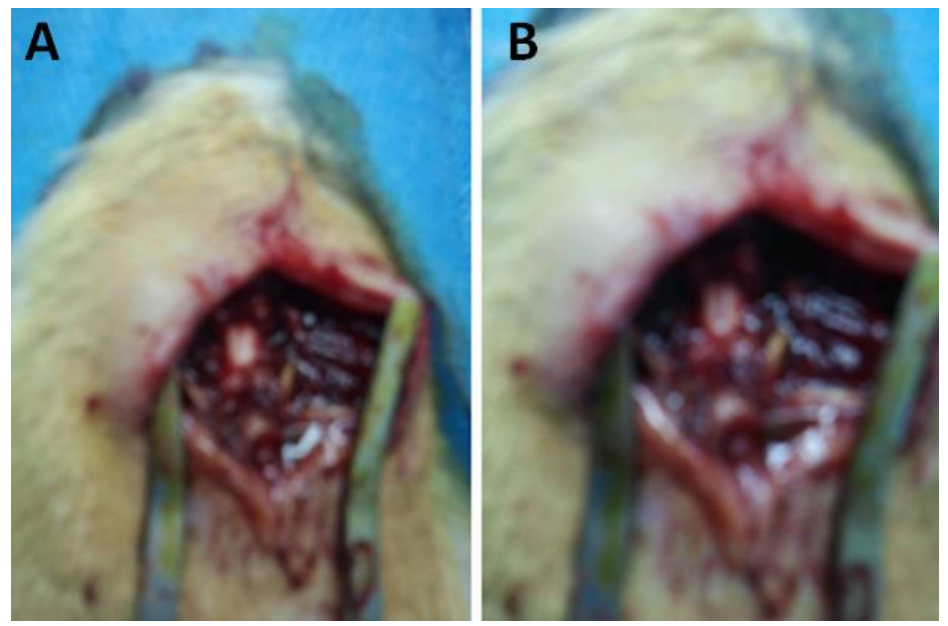

Figure 1. Laminectomy (A) and creating spinal cord injury after laminectomy with dropping ten-gram weight from a height of $25 \mathrm{~mm}$ on the spinal cord in the T10 segment of the spinal cord (B), shiny appearance of spinal cord demonstrates intact Dura matter.

After the injury, muscles and skin of the site were sutured, in order to prevent dehydration, $10 \mathrm{mg}$ of Ringer's solution was injected intraperitoneally to the animal. Cefazolin $(10 \mathrm{mg} / \mathrm{kg})$ was also injected until two days after surgery. Bladder emptying was performed twice a day until establishing a urinary reflex. After the creation of injury and consciousness of the animals, BBB motor test was performed for two days, and animals that have BBB score higher than three $48 \mathrm{~h}$ after injury were excluded from the study. And animals with a score of less than three were assessed until the end of the study (twelve weeks).

\section{Functional assessment by BBB motor} test: In order to assess the motor function of the animals, BBB (Basso, Bregnahan and Beattie) test was used (21). In this test, scores of zero and 21 mean lack of motion and normal motion of the animal, respectively .

Animals with a score of 3 or higher in the first 48 hours after injury were excluded from the behavioral study and animals with a score of less than 3 were selected for the rest of the study.

The test was carried out on all animals within 48 hours after the injury on a daily basis and then on a weekly basis (once a week) for a period of twelve weeks by two individuals separately and the final score was reported as an average of the given scores .

\section{Statistical analysis}

Statistical analysis was performed using the Minitab 16 statistical software. Data was reported as mean \pm SD at significance level of $\mathrm{P}<0.05$. Differences between the groups were reported significant when $\mathrm{P}<0.05$. Comparisons between groups using one-way ANOVA with the significance level of $\mathrm{P}<0.05$ was performed.

\section{Results}

Comparing the BBB test results between the groups throughout the study indicate a significant difference between the normal control and sham groups with the injury group $(\mathrm{P}<0.05)$ (Figure 2).

Comparing the motor test results of normal control and sham-operated groups from the beginning to the end of the study (twelve weeks) showed no significant difference $(\mathrm{P}>0.05)$, which indicates that laminectomy alone does not impair motor function in animals. However, there is a significant difference between both normal control and sham groups with injury group $(\mathrm{P}<0.05)$ (Figure 2).

As the figure 2 shows in the group with injury at the end of the second week to the 
fifth week of the study a motor recovery has been observed with a slow trend and the amount increased at a faster rate after the fifth week until the end of the study, so that by the end of the study (week 12) motor score of the animals of the group with injury reached 6.5 that represents a spontaneous relative recovery in animal without the intervention.

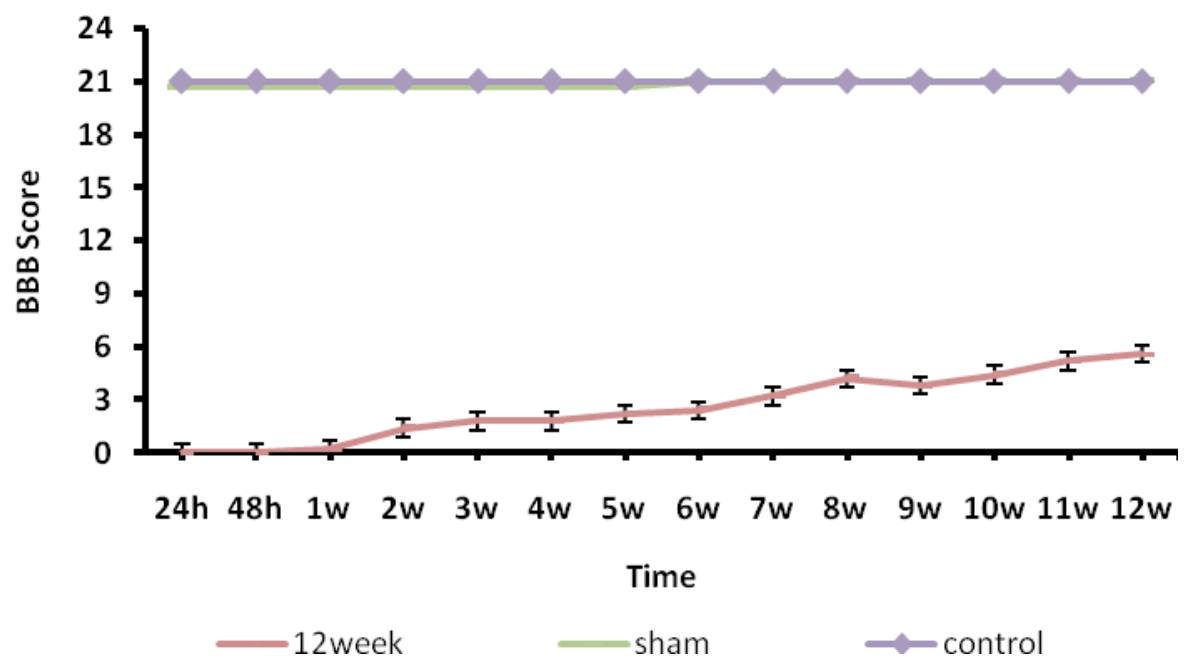

Figure 2. Functional recovery assessment of animals in the groups studied until the end of the 12th week. Results are shown as mean \pm SD and statistically significant difference has been expressed in the level of $P<0.05$. As the graph shows a relative functional recovery observes as the motor test score of animals from 1.4 in the second week after the injury reached to 6.5 in the twelfth week.

\section{Discussion}

In the present study, the spinal cord injury of severe contusion type at T10 spinal cord segment was created and then motor function was evaluated for twelve weeks without any medical intervention and the results showed that over time a relative recovery in the movement of animals may be achieved.

At the end of the study that was performed by Verdu et al in 2003 on spinal cord injury of photochemical type (halogen lamp shining on the exposed spinal cord for 2.5 minutes), BBB scores of the injury group without intervention in the first week was 11 and from the second week to the end of the third month (twelve weeks) was 18 (18). Also in a study by GarciaAlias et al (2004) on acute photochemical spinal injury (30 minutes after the injury) it was shown that the BBB score of the injury without the intervention group at the end of the first week and at the end of study (the week twelve) was 11 and 15, respectively(19). However, in our study the BBB scores at the end of week twelve was 6.5, which is the reason for this difference may be due to differences in the severity and the site of the injury into the spinal cord because the severity of the photochemical injury used in the study by Verdu and Garcia-Alias is much more mild than that of the contusion injury in our study.

On the other hand, in a study by Fouad et al (2005) after complete injury of adult rat spinal cord at T7-9, the mean motor score of the injury without the intervention group was reported 2.1 showing lower motor recovery in comparison with the case group of our study (23). That may be due to the more severe injury (complete cessation of the spinal cord) in this study. Richter et al (2005) in their study on spinal cord injury of posterior funiculus scratch 
type reported a limited regeneration in the injury without intervention group due to weak internal capacity of neurons for reconstruction or resulting from angiogenesis (20).

Mothe and Tator (2005) stated that type of cells stimulated in response to injury is different depending on the model of injury. In the transection model of the spinal cord responses of stem/progenitor cells are locally limited to the area of the injury, while in contusion and compression models the responses of these cells are systemic mitotic responses that can be seen throughout the rostrocaudal part of spinal cord, the broader response is probably because larger areas of spinal cord tissue are affected in the area of the injury.

It was recently shown that some stem cells and progenitor cells present in ependymal canal may be called and recover damage with increased proliferation (9). However, the cell proliferation may not always lead to the restoration of spinal cord function and sometimes only fills the cavity (7).

Therefore, according to the results it

\section{References}

1. Beattie MS, Hermann GE, Rogers RC, Bresnahan JC. Cell death in models of spinal cord injury. Prog Brain Res. 2002; 137: 37-47.

2. Sie I, Waters R. Outcomes following spinal cord injury. In: Lin V, editor. Spinal cord medicine: principles and practice. New York: Demos Medical Publishing; 2003. p. 87-103.

3. Okada S, Nakamura $M$, Katoh $H$, Miyao T, Shimazaki T, Ishii K, et al. Conditional ablation of State 3 or Socs 3 discloses a dual role for reactive astrocytes after spinal cord injury. Nat Med. 2006; 12(7): 829-34.

4. Kundi S, Bicknell R, Ahmed Z. Spinal cord injury: Current mammalian models. Am J Neurosci. 2013; 4 (1):112. appears that the relative functional recovery observed in this study is due to calling of stem and progenitor cells at the site of the injury, poor regeneration of neurons at the site, angiogenesis, even reorganization of intact circuits and plasticity change.

\section{Conclusion}

Due to time-dependent increase in BBB score of rats with spinal cord injury it seems that the animals also have a relative functional recovery with no therapeutic interventions. However, in order to determine the maximum level of recovery, duration of the study is the need to be increased.

\section{Acknowledgment}

The authors of this paper would like to appreciate the efforts of all those who helped to carry out this study, especially Mr. Basereh at Laboratory Animals Care Center.

5. Young W. Spinal cord contusion models. Prog Brain Res. 2002; 137:231-255.

6. Tator $\mathrm{CH}$. Update on the pathophysiology and pathology of acute spinal cord injury. Brain Pathol.1995; 5(4): 407-13.

7. Kostyk S, Popovich P, Stokes B. Mechanisms and natural history of spinal cord injury. In: Lin V, editor. Spinal cord medicine: principles and practice. New York: Demos Medical Publishing; 2003. p. 765-76.

8. Oudega M, Moon LD, de Almeida Leme RJ. Schwann cells for spinal cord repair. Braz J Med Biol Res. 2005; 38(6):825-35.

9. Moreno-Manzano V, RodriguezJimenez F, Garcia-Rosello M, Lainez S, Erceg S, Calvo M, et al. Activated spinal cord ependymal stem cells 
rescue neurological function. Stem Cell. 2009; 27(3):733-43.

10. Mothe AJ, Tator CH. Proliferation, migration, and differentiation of endogenous ependymal region stem/ progenitor cells following minimal spinal cord injury in the adult Rat. Neuroscience. 2005; 131(1): 177-87.

11. McDonough A, Martinez-Cerdeno V. Endogenous proliferation after spinal cord injury in animal models. Stem Cells Int. 2012; 2012:387513.

12. Filbin MT. Axon regeneration, vaccinating against spinal cord injury. Curr Biol. 2000; 10(3): R100-R103.

13. De Winter F, Oudega M, Lankhorst AJ, Hamers FP, Blits B, Ruitenberg $\mathrm{MJ}$, et al. Injury -induced class 3 semaphorin expression in the rat spinal cord. Exp Neurol. 2002; 175(1): 61-75.

14. Properzi F, Carulli D, Asher RA, Muir E, Camargo LM, Kuppevelt THV, et al. Chondroitin 6-sulphate synthesis is up-regulated in injured CNS, induced by injury related cytokines and enhanced in axon-growth inhibitory glia. Eur J Neurosci. 2005; 21(2):37890.

15. Yaojian R, Wenxiao Z, Huijuan L, Chunxia S, Qingan Z, Yongjun W, et al. Clinical application of olfactory ensheathing cells in the treatment of spinal cord injury. J Int Med Res. 2013; 41(2): 473-81.

16. Fawcett JW, Asher RA. The glial scar and central nervous system repair. Brain Res Bull. 1999; 49(6): 377-91.

17. Ueno M, Yamashita T. Strategies for regenerating injured axons after spinal cord injury - insights from brain development. Tar Ther. 2008; 2(2):253-64.
18. Verdu E, Garcia - Alias G, Joaquimfore S, Lopez -Vales R, Navarro $\mathrm{X}$. Olfactory ensheating cells transplanted in lesion spinal cord prevent loss of spinal cord parenchyma and promote functional recovery. Glia. 2003; 42(3): 275-86.

19. Garcia-Alias G, Lopez-Vales R, Fores $J$, Navarro $X$, Verdue E. Acute transplantation of olfactory ensheating cells or schwann cells promotes recovery after spinal cord injury in the rat. J Neurosci Res. 2004; 75(5):63241.

20. Richter MW, Fletcher PA, Liu J, Tetzlaff W, Roskams AJ. Lamina propria and olfactory bulb ensheathing cells exhibit differential integration and migration and promote differential axon sprouting in the lesioned spinal cord. Neuroscience. 2005; 25(46):10700-11.

21. Basso DM, Beattie MS, Bresnahan JC. Graded histological locomotor outcomes after spinal cord contusion using the NYU weight-drop device versus transaction. Exp Neurol.1996; 139(2):244-56.

22. Wang C, Chen J, Wu Y, Tsai M, Shyue S, Yang C, et al. Reduction in antioxidant enzyme expression and sustained inflammation enhance tissue damage in the subacute phase of spinal cord contusive injury. J Biomed Sci. 2011; 18(1): 13.

23. Fouad K, Schnell L, Bunge MB, Schweb ME, Liebscher T, Pearse DD. Combining schwann cell bridges and olfactory ensheathing glia grafts with chondroitinase promotes locomotor recovery after complete transection of the spinal cord. Neuroscience. 2005; 25(5):1169-78. 